\title{
Glycogen-rich adenocarcinoma in the lower lip: report of a case with particular emphasis on differential diagnosis
}

\author{
Kazumasa Mori", Jun Shimada, Nozomi Tamura, Nobuaki Tamura \\ Division of First Oral and Maxillofacial Surgery, Department of Diagnosis and Therapeutics Sciences, \\ Meikai University School of Dentistry, Saitama, Japan. \\ Email: *kazu-mori@dent.meikai.ac.jp
}

Received 22 June 2011; revised 25 July 2011; accepted 9 August 2011.

\begin{abstract}
We herein report a glycogen-rich adenocarcinoma occurring in the lower lip of a 44-year-old female. The lesion appeared as painless slowly growing mass in the right side of the lower lip. Histologically, infiltrative tumor nests with duct-like or pseudocyst structure into the mucosa and/or dermis were observed. The basaloid neoplastic cells had granular but occasionally vacuolated cytoplasm with salient reaction of diastase-digestible periodic acid-Schiff, thus indicating that they were glycogen-rich in nature. The tumor cells were positive for CEA, cytokeratin, and S-100 protein, and negative for SMA, alpha-1 antitrypsin, and amylase by immunohistochemistry. The origin of the tumor with infiltrative nests was indistinguishable from salivary gland and skin appendage malignancy. A differential diagnosis of this peculiar neoplasm was discussed.
\end{abstract}

Keywords: Adenocarcinoma; Glycogen; Differntial Diagnosis; Lower Lip

\section{CASE PRESENTATION}

A 44-year-old female was referred to the 1st Department of Oral and Maxillofacial Surgery by a dentist for diagnosis and management of a painless "lump" on the right side of her lower lip. The mass had been noticed 8 years before the current consultation. According to the patient, the mass has recently enlarged, interfering with her mastication, but caused no difficulty in speech. No changes of size or growth of the mass during meal times were reported. The patient was in apparent good health and her medical history was noncontributory.

Clinical examination showed a $2.5 \times 2.5 \mathrm{~cm}$ swelling with induration on the right side of the lower lip (Figure 1). It was covered by skin that was normal in both texture and color. Intraorally, a whitish lesion with a papillary outgrowth (Figure 2), in which there was a partial ulcerated surface, was observed. No induration or pain was detected in the regional lymph node. A computerized tomography (CT) scan confirmed a tumor-like mass with an unclear border in the lower lip (Figure 3). Magnetic resonance imaging (MRI) showed a high signal in the tumor-like mass in a T2 weight image (Figure 4).

\section{DIFFERENTIAL DIAGNOSIS}

The lower lip may be affected by several pathologic conditions, including inflammatory, obstructive, traumatic, or neoplastic causes [1,2]. Lesions in this area may be present for a prolonged period of time before the patient seeks a medico-dental consultation, usually as a result of speech or cosmetic insufficiency.

Inflammatory disorders of the minor salivary gland were considered for the differential diagnosis of the present lesion. An acute sialadenitis of the lower lip usually represents as a swelling associated with pain and redness, and chronic sialadenitis runs a prolonged course of post-inflammatory sclerosis.

Mucous retention phenomenon or a mucous retention cyst can affect any minor salivary gland site intraorally, but is most frequently encountered in the lower lip of young individuals. They are characterized by a soft fluctuant swelling that may present bluish dome-like nodules. The lesion is usually treated by conservative surgical excision. Because of its benign nature, the lesion does not have any tendency to recur.

Dermoid and/or epidermoid cyst was an other possible lesion that may occur in the lower lip as well. Dermoid cysts are most commonly seen in the midline of the floor of the mouth, while epidermoid cysts are common in acne-prone areas of skin on the face, neck and back. However these cystic lesions are classified as a developmental cyst, and young adults are more likely to be affected.

Salivary gland tumors including monomorphic adenoma and benign mixed tumors (MT), commonly present in the third to sixth decades, should be differentiated, 


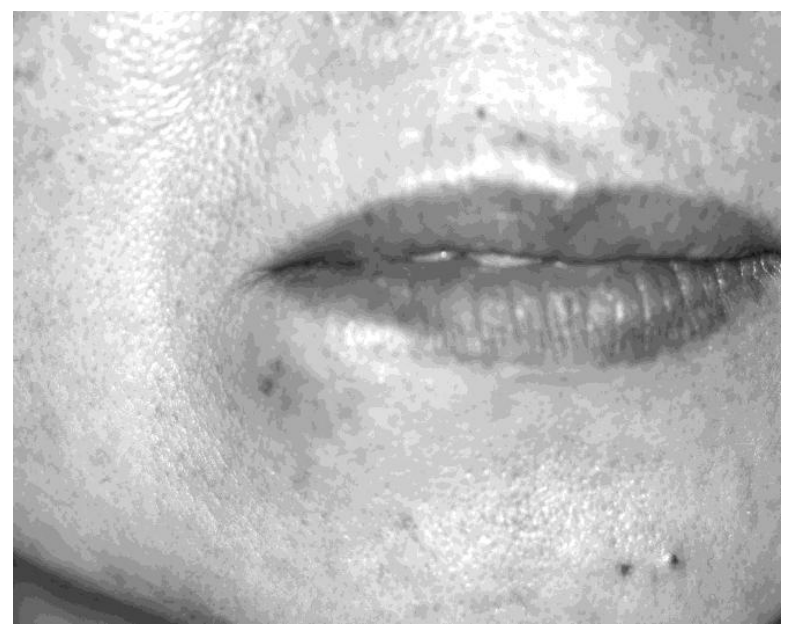

Figure 1. Extra oral view at first visited, showing swelling on the right side of the lower lip.

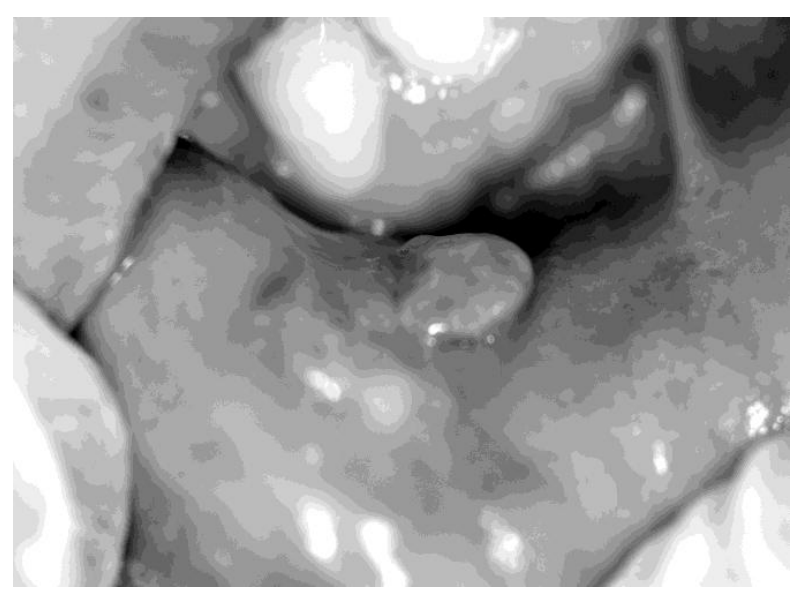

Figure 2. A whitish lesion with papillary outgrowth on the inner side of the lip.

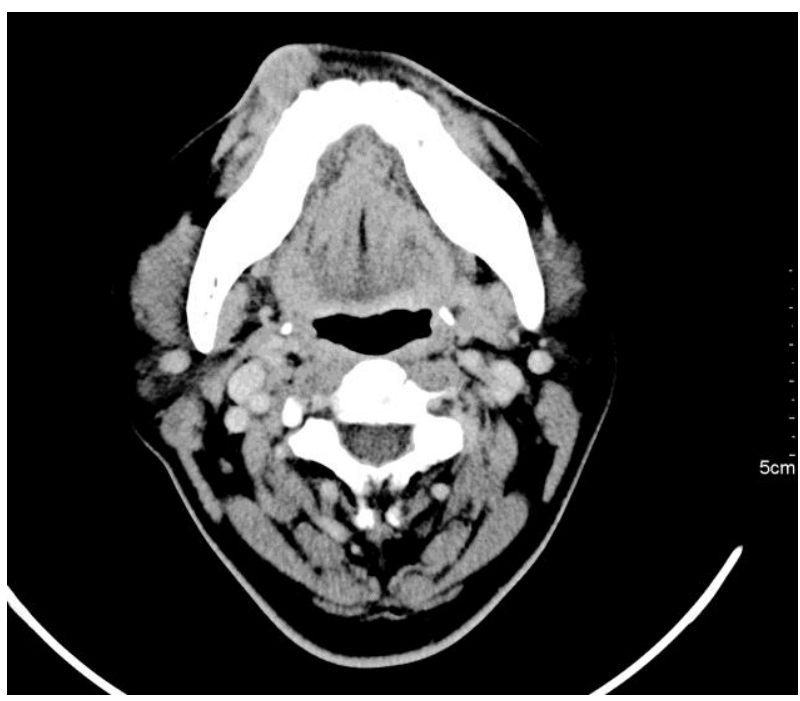

Figure 3. The CT findings revealed a tumor with an unclear border in the lower lip.

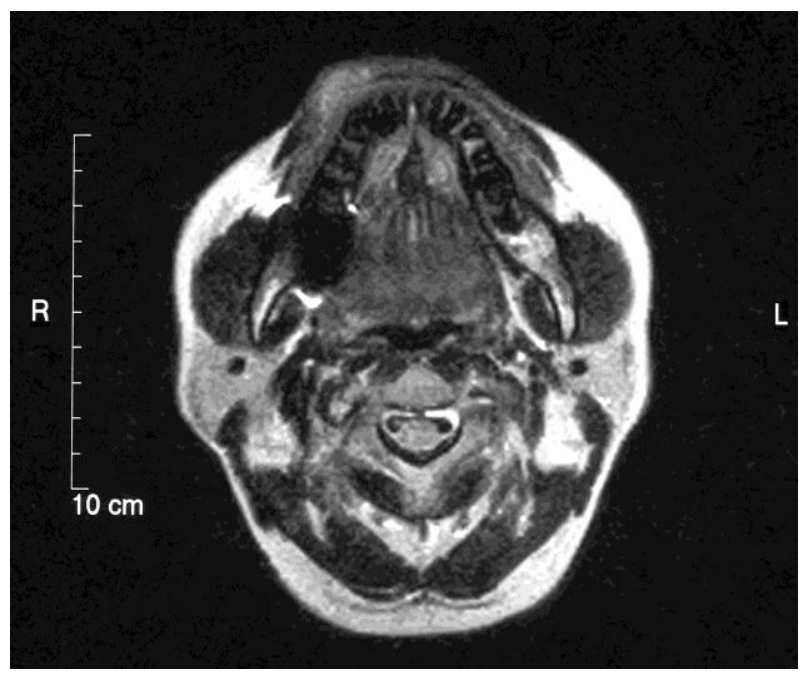

Figure 4. MRI findings revealed a high signal in the tumor mass in a T2 weighted image (T2 weighted MRI image).

as they may rarely arise in the lower lip [3]. Monomorphic adenomas are usually encapsulated or well circumscribed. MTs are partially circumscribed, exhibiting the characteristic histologic featurers with myxoid and chondroid areas. They generally have more varied growth pattern consisting mostly of myoepithelial cell proliferation.

Because of its frequency among the salivary gland tumors, a diagnosis of MT should be approached with caution to exclude other salivary gland neoplasms with malignant features, mucoepidermoid and adenoid cystic (ACC) carcinomas. Although these tumors do not always show a rapid growth pattern or even cytologic atypia, their microscopic characteristics can readily be distinguished.

Cutaneous skin appendage tumors [4-6], including microcystic adnexal carcinoma, porocarcinoma, and adenoid cystic carcinoma of the skin (DACC) were also considered. Among of these skin malignancies, DACCs are morphologically similar to those arising in salivary glands, and are composed of a somewhat uniform population of basaloid tumor cells. However, malignant tumors of the skin genellary appear as a pedunculated mass or nodule on the skin with a rapid growth pattern.

Before the diagnosis of a primary submucosal or cutaneous malignant tumor is made, the possibility of a metastasis from other organs, such as from the breast [7], should be ruled out based on the clinico-pathological background. It should be emphasized, therefore, that careful examination of the breasts and other organs, in combination with meticulous history-taking, are necessary to make an accurate differential diagnosis.

\section{DIAGNOSIS AND TREATMENT}

Light microscopic examination of hematoxylin-eosin- 
stained sections of the tumor revealed closely apposed neoplastic nests in the submucosal and/or subcutaneous region with an infiltrative growth pattern. The tumor was composed of basaloid cells forming small duct-like spaces (or pseudocysts) within the tumor nests (Figure 5). Nuclear hyperchromatisum and nuclear disarrangement were subtle, and only rare mitotic figures were present.

Routine hematoxylin and eosin histologic features were insufficient to make a definitive diagnosis. Therefore, we performed periodic acid-Schiff (PAS) and PAS after diastase digestion (D-PAS). The D-PAS revealed an apparent disappearance of the conventional PAS reactive material (Figures 6 (a) and (b)). These results indicated an accumulation of glycogen in their cytoplasm, possibly derived either from the minor salivary gland or skin appendage organs. However, these results alone did not provide enough evidence to distinguish between a salivary gland tumor and a skin appendage tumor, since glycogen-rich characteristics can be seen both in salivary gland tumors and in some skin appendage tumors [4].

Immunohistochemically, the tumor cells showed a positive reaction for cytokeratin (Figure 7), S-100 protein (not shown), CEA in part (Figure 8), but a negative reaction for smooth muscle actin (SMA), alpha-1 antitrypsin, and amylase. The positive result for the S-100 protein indicated a possibility of myoepithelial origin. However, the negative reaction for SMA excluded the possibility that the tumor was myoepithelial in nature.

As described, the possibility of skin appendage malignancy, such as microcystic adnexal carcinoma or eccrine porocarcinoma [5], was considered in the differential diagnosis of the present tumor. However, in consideration of the histologic, histochemical, and immunohistochemical findings, the final diagnosis was tentatively made as a glycogen-rich adenocarcinoma of undetermined origin.

After the diagnosis of the lower lip malignancy was made by the biopsy specimen, the patient was transferred to the Saitama Prefectural Cancer Institute where the lesion was surgically removed. Seven months after the primary surgery, a swelling was observed in the right submental lymph node, and lymphectomy was performed. Post-surgical lymph node specimens exhibited a metastatic focus of the tumor, with extra-capsular invasion.

During the follow-up period (approximately ten months) after the lymphectomy, further swelling was noted in the right submandibular lymph nodes, and radical neck dissection was performed. A metastatic tumor focus was again observed in the histlogical sections. However, neither recurrence nor metastatic signs have been present since the neck surgery. The patient's post-surgical status has been uneventful during the past five years.

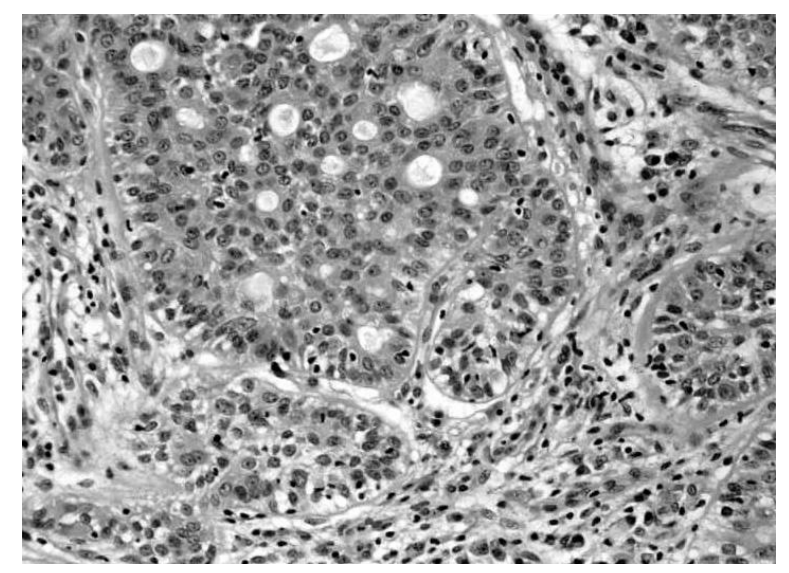

Figure 5. A small duct-like or pseudo cystic structures were detected in tumor nests (hematoxylin and eosin, original magnification, $\times 200$ ).

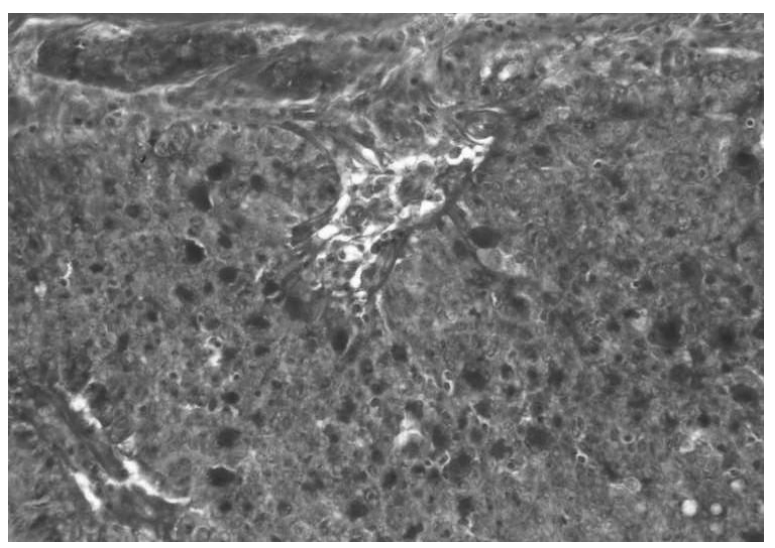

(a)

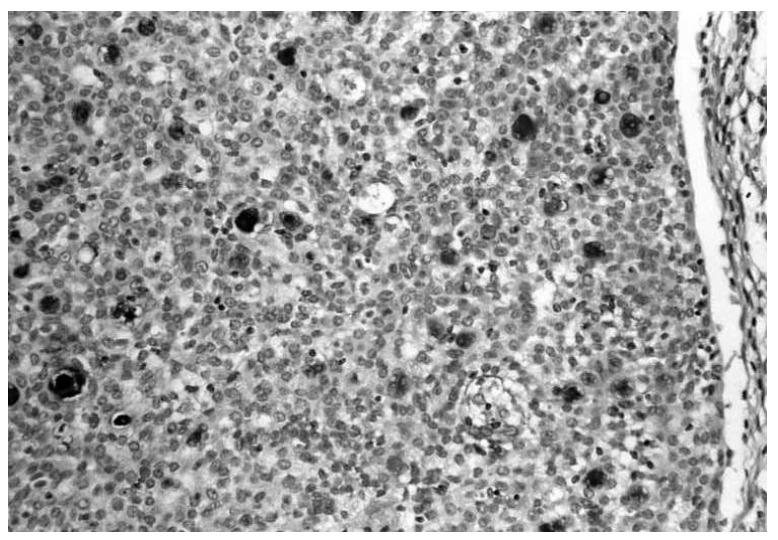

(b)

Figure 6. (a) Positive reaction to PAS stain in the tumor nests and pseudocysts (PAS stain, original magnification, $\times 200$ ); (b) Disappearance of PAS-positive material after diastase digestion seen in the tumor nests (PAS stain after diastase digestion, original magnification, $\times 200)$. 




Figure 7. Immunohistochemical detection of cytokeratin in the tumor nests (original magnification, $\times 200$ ).



Figure 8. Immunohistochemical detection of CEA the tumor nests (original magnification, $\times 200$ ).

\section{DISCUSSION}

Intraoral glycogen-rich adenocarcinoma is a rare neoplasm particulary in the lower lip. The accurate characteristics of the cells of origin have not yet been fully clarified. In the head and neck region, tumors of salivary gland origin [1-3], dermal appendage derivation [4-6], and metastatic tumors from a distant organ (such as the breast) [7] should be included in the differential diagnosis. In our case, metastatic malignancy was ruled out by careful systemic examinations, using conventional X-ray, CT, MRI, and even by blood biochemistry.

Before considering these other diseases, the first thought should be that the tumors is a primary salivary gland malignancy, such as an ACC or mucoepidermoid carcinoma. In particular, the cribriform of ACC, which is composed of sheets of basaloid cells forming "cribriform” pseudocysts within the tumor nests, may show a similar cytopathology to the present case. However, the possibility of ACC and mucoepidermoid carcinoma was excluded by the abundant accumulation of glycogen within the tumor nests in the present case.

The published literature about glycogen-rich or clear cell carcinoma of the salivary gland have described it as epithelial-myoepithelial carcinoma [3]. This tumor manifests a biphasic growth pattern consisting of the small ductal cells that are surrounded by the proliferating myoepithelial clear cells. Immunohistochemically, the ductal cells show cytokeratin, while the clear cells demonstrate strongly positive immunoreactions for S-100 protein and SMA, and a variable degree of staining for cytokeratin. Again, although some amount of glycogen can be seen in clear cells, epithelial-myoepithelial carcinoma could be excluded by the large amount of glycogen accumulation seen in the present case.

The accumulation of glycogen suggests the possibility of a malignant tumor of a cutaneous skin appendage. The presence of glycogen in most of the tumor cells in the present case could be consistent with their being immature cells of the intraepidermal eccrine sweat gland duct, since these cells are rich in glycogen in the human embryo [4]. Therefore, skin appendage tumors shoud be differentiated from primary salivary gland tumors, and skin lesions arising near the salivary gland may provide diagnostic difficulty. A more real concern is the distinction between glycogen-rich adenocarcinoma and eccrine adenocarcinoma, both of which are rich in cytoplasmic accumulation of glycogen.

The treatment of choice for the lower lip malignancy is surgical excision, together with some kind of chemotherapy. Our patient was treated with surgical excision with a $10 \mathrm{~mm}$ safety margin. Seven months after the surgery, a suspicious metastatic focus was found in her regional cervical lymph nodes. The regional lymph nodes were removed, and the focus was diagnosed as a metastatic tumor of the primary carcinoma. A relatively good prognosis was obtained, and she has been recurrence and metastasis-free for about five years after the radical neck surgery. However, since local recurrence and distant metastasis are possible because of the malignant nature of the tumor, long-term careful follow-up is needed.

\section{AKNOWLEDGEMENTS}

The authors express their gratitude to Drs. S. Okabe and K. Yagihara, Saitama Prefectural Institute of Cancer, for their helpful discussion and management of the patient in due of follow-up course, and Dr. Y. Tajima, Department of Oral Pathology, Meikai University School of Dentistry, for preparing this manuscript.

\section{REFERENCES}

[1] Gnepp, D.R. (2001) Diagnostic surgical pathology of the head and neck. W.B. Saunders, Philadelphia, 325-430.

[2] Neville, B., Damm, D.D., Allen, C.M. and Bouquot, J.E. 
(2009) Oral and maxillofacial pathology. 3rd Edition, Saunders, Elsevier, 473-506.

[3] Ellis, G.L., Aauclair, P.L. and Gnepp, D.R. (1991) Surgical pathology of the salivary glands. W.B. Saunders, Philadelphia, 135-164, 412-421.

[4] Elder, D.E., Elenitsas, R., Johnson, B.L. and Murphy, G.F. (2008) Lever's histopathology of the skin. 10th Edition, Lippincott Williams \& Wilkins, 851-910.

[5] LeBoit, P.E., Burg, G., Weedon, D. and Sarasin, A. (2006) Pathology and genetics of skin tumours. World Health
Organization Classification of Tumours, 125-138.

[6] Rutten, A., Requena, L. and Requena, C. (2002) Clear cell porocarcinoma in situ: A cytologic variant of porocarcinoma in situ. The American Journal of Dermatopathology, 24, 67-71.

doi:10.1097/00000372-200202000-00014

[7] Trupiano, J.K., Ogrodowczyk, E. and Bergman, S. (2003) Pathologic quiz: Mass in the right breast. Archives of $\mathrm{Pa}$ thology \& Laboratory Medicine, 127, 1629-1630. 